\title{
Childhood Central Nervous System Ganglioneuroblastoma
}

National Cancer Institute

\section{Source}

National Cancer Institute. Childhood Central Nervous System Ganglioneuroblastoma.

NCI Thesaurus. Code C124272.

A cerebral gang lioneuroblastoma that occurs during childhood. 